\title{
Searching the Footprint of WIMPZILLAs
}

\author{
Houri Ziaeepour \\ ESO, Schwarzchildstrasse 2, 85748, Garching b. München, Germany \\ Email: houri@eso.org
}

\begin{abstract}
We constrain mass, lifetime and contribution of a very slowly decaying Ultra Heavy Dark Matter (UHDM) by simulating the cosmological evolution of its remnants. Most of interactions which participate in energy dissipation are included in the numerical solution of the Boltzmann equation. Cross-sections are calculated either analytically or by using PYTHIA Monte Carlo program. This paper describes in detail our simulation. To show the importance of the distribution of matter in constraining WIMPZILLA [1] characteristics, we consider two extreme cases: a homogeneous universe, and a local halo with uniform distribution. In a homogeneous universe, the decay of the UHDM with a mass $\sim 10^{15} \mathrm{GeV}$ and a lifetime as short as a few times of the age of the Universe, can not explain the flux of the observed Ultra High Energy Cosmic Rays (UHECRs) even if the whole Dark Matter (DM) is composed of a decaying UHDM. If our simple halo model is reliable, in a uniform clump with an overdensity of $\sim 200$ extended to $\sim 100 \mathrm{kpc}$, the lifetime can be $\sim 10-100 \tau_{0}$, again assuming that DM is a decaying UHDM. We also compare our calculation with observed $\gamma$-rays at $E \sim 10^{11} \mathrm{eV}$ by EGRET and CASA-MIA limit at $E \sim 10^{15} \mathrm{eV}$. They are compatible with a UHDM with relatively short lifetime.
\end{abstract}

PACS codes: 95.35.+d, 98.70.Sa, 13.85.Tp, 98.70.Vc, 95.30.Cq, 04.25.Dm

\footnotetext{
${ }^{1}$ Present Address: 03, impasse de la Grande Boucherie, F-67000, Strasbourg, France.
} 


\section{First Encounter}

Particle Physics today is a zoo with plenty of wild species very difficult to trace or capture. They are in general known under phyla WIMPs, LSP, Axions, Higgs, Heavy Neutrinos, etc. Most of these species are potential candidates of the Dark Matter.

Recently a new phylum called WIMPZILLA [2] has been added to this zoo. The only common characteristic of the members of this family is their enormous mass, close to GUT scale $10^{16} \mathrm{GeV}$ and their presumed long lifetime, much larger than the age of the Universe.

Theoretical motivations for existence of these particles is not very new. Since early 90s, some compactification scenarios in string theory have predicted composite particles (e.g cryptons) with large symmetry groups [3] and $M \gtrsim 10^{14} \mathrm{GeV}$. M-theory [⿶凵⿴囗十 provides better candidates if compactification scale is much larger than Standard Model weak interaction scale [5]. Messenger bosons in soft supersymmetry breaking models (see [6] for review) also can have close to GUT scale masses. Being composite and decaying only through non-renormalizable interactions or having discrete gauge symmetry [7] can make these ultra heavy particles meta-stable. Parametric resonance [8] or vacuum fluctuation [2] at the end of inflation can produce a large amount of UHDM and unitarity constraint on their mass [9] can be overcome if they have never been thermalized [2].

If there was not other motivation for existence of an ultra heavy meta-stable particle, it was just one of many predictions of Particle Physics models waiting for detection. However, the discovery of Ultra High Energy Cosmic Rays (UHECRs) by large Air Shower detectors [10] [11] [12] (For a review of UHECRs detection and observed properties see [13]) is an observational motivation. The predicted GZK cutoff [14] in the spectrum of CRs at energies around $\sim 10^{18} \mathrm{eV}$ due to interaction with CMBR and IR photons restricts the distance to sources to less than $20-30 M p c$. At present there are about 600 events with $E \sim 10^{19} \mathrm{eV}, 50$ with $E>4 \times 10^{19} \mathrm{eV}$ and 14 events with $E>10^{20} \mathrm{eV}$ [15] [16] including one with $E \sim 10^{21} \mathrm{eV}$ [11]. The UHECR spectrum has a local minimum around $E \sim 10^{19} \mathrm{eV}$ but it rises again at higher energies.

Composition of the primary particles [17] [18] can be estimated from the shower and its muon content maximum position and their elongation rate in the atmosphere. In spite of uncertainties and dependence on hadrons interaction models at high energies [19], all analyses of the data are compatible with a composition change from iron nuclei to proton at $E>10^{18} \mathrm{eV}$ [11] [17] [18] [20]. Based on theoretical arguments however, some authors suggest that events with highest energies can be produced by heavy nuclei [21] (But see also [20] for maximum fly distance of Fe nuclei).

Some of ideas about the origin of UHECRs has been reviewed in [22] and references therein. For the sake of completeness here we briefly review arguments for and against conventional and 
exotic sources.

Classical Candidates: In a recent review of conventional candidates of UHECR sources [23], R. Blandford rules out practically all of them.

It is expected that charged particles are accelerated in the shock waves of AGNs, SNs, or infalling gas in rich galaxy clusters. Their maximum energy is proportional to the magnetic field strength and the size of the accelerator. Using this simple estimation, remnants of old SNs are expected to accelerate protons up to $\sim 10^{15} \mathrm{eV}$ and iron to $\sim 10^{18} \mathrm{eV}$ [24]. A $\sim 100 \mathrm{TeV} \gamma$-ray is expected from synchrotron radiation of these protons but nothing has been observed [25]. Shock front of AGNs relativistic jets can accelerate protons to $E \sim 10^{20} \mathrm{eV}$ [26]. Central black hole in Fannaroff-Riley galaxies [27] or the remnant of QSO [28] can produce UHECRs up to $E \sim 10^{20} \mathrm{eV}$ with a marginal maximum energy of $4 \times 10^{21} \mathrm{eV}$ [29]. Adiabatic expansion [30] and in situ interaction with radiation and matter fields however reduce the achievable maximum energy by orders of magnitude. Abrupt termination of the acceleration zone or composition change from $p$ to $n$ [30] increases the chance of particles to keep their energies. The former however needs a fine tuning of the source structure and in the latter case particles lose part of their energy anyway.

Gamma Ray Bursts (GBR) also have been proposed as a candidate source of UHECRs [31]. A simulation [32] of cosmological distribution of sources with a power law flux of UHECRs shows however that the expected flux on Earth is much lower than observed value.

In 33] it is argued that Poisson noise in the process of proton interaction with background photons leaves a non-interacting tail in the flux of UHECRs and increases the probability of detecting UHECRs from further distances. Optical depth of protons around GZK cutoff can be roughly estimated by $\tau_{o p t} \approx \sigma n_{c m b}$. For $\sigma \approx 0.45 \mathrm{mb}$ close to the resonance, the probability of non-interacting in a distance of $30 M p c$ is at most $\sim 10^{-8}$.

Correlation with Astronomical Objects: At present no serious astronomical candidate source has been observed. A correlation between Super Galactic Plane and UHECR events direction has been claimed [38], but ruled out by other analyses [39]. It is plausible that the apparent clustering of events reported by AGASA collaboration 15 originates from caustics generated by the galactic magnetic field [36] [37] [34] [40].

Most of UHECRs burst models predict only one important nearby source [31] [33]. M87 in Virgo Cluster is practically the only conventional candidate that respects the distance constraint and is probably able to accelerate protons to ultra high energies. Recently, it has been shown that galactic wind and its induced magnetic field can deflect protons with $E>10^{20} \mathrm{eV}$ if the magnetic field is as high as $7 \mu G$. In this case, most of UHECR events point to Virgo Cluster [40]. Even with such strong magnetic field, this model can correlate most energetic events with M87/Virgo 
only if the primaries are $H e$ nuclei.

There are also claims of correlation between the direction of UHECRs and pulsars 41] or QSOs [42]. The latter is reliable only if ultra high energy $\nu$ s produce protons by interacting with relic or a halo of $\nu$ [43]. Recent works [44] however rule out a large part of the parameter space.

Close to uniform distribution of UHECRs events has been concluded to be the evidence that UHECRs originate from some extra-galactic astronomical objects and not from a decaying UHDM in the Galactic Halo [45] [46] [47]. MACHOs observation [84 however shows that the Halo has a heterogeneous composition and a precise modeling of the anisotropy must take into account the distribution of various components.

Exotic Sources: Ultra Heavy particles can be either long life DM or short life particles produced by the decay of topological defects [50]. In the latter case, the heavy particles decay in their turn to ordinary species and make the observed UHECRs. The result of the decay of a heavy short life particle and a heavy relic can be quite the same, but their production rate and its cosmological evolution are very different and depend on the defect type and model [51]. The possibility that topological defects be the source of UHECRs has been studied extensively [51] [59] [22]. Nonetheless, the observed power spectrum of LSS and CMB anisotropies rule out the existence of large amount of defects in the early Universe [53] [54 and consequently the chance that UHECRs be produced by their decay (there are also limits from high energy $\nu$ 22]).

Another proposed source of UHECRs is the evaporation of primordial black holes (PBH). The Hawking temperature at the end of their life is enough high to produce extremely energetic elementary particles like quarks and gluons and thus UHECRs. Most of models for production of PBH however needs fine tuning. Moreover, they are mainly produced when a large over density region crosses the horizon [55]. PBHs with present temperature of the same order as UHECRs energy must have an initial mass of $10^{14}-10^{15} \mathrm{gr}$ and thus formed when the temperature of the Universe was $\sim 10^{9} \mathrm{GeV}$. A thermal inflation [56] at EW scale would have reduced their density $\sim 10^{12}$ times. In models with low reheating temperature, at these scales the Universe is not yet thermalized and parametric resonance and fluctuation production happens only at superhorizon scales [8].

Summarizing the discussion of this section, it seems that conventional sources of Cosmic Rays are not able to explain the observed rate of UHECRs [23]. Between exotic sources, the decay of a meta-stable ultra heavy particle seems to be the most plausible one. 


\section{WIMPZILLA Decay and Energy Dissipation of Remnants}

The decay of UHDM can have important implications for the evolution of high energy backgrounds. This can also be used for verifying this hypothesis and constraining the mass and lifetime of these particles. A number of authors have already tried to estimate the possible range of parameters as well as the flux of remnants on Earth (see [57] and [59] for defects, and [58], 60], 61] and 62] for UHDM). In the present work more interactions have been included in the energy dissipation of UHDM remnants. Hadronization is implemented using PYTHIA Monte Carlo program 63. We consider two distributions for UHDM. First we study the evolution of the spectrum of stable particles i.e. $e^{ \pm}, p^{ \pm}, \nu, \bar{\nu}$ and $\gamma$ in a homogeneous universe from photon decoupling to today. Then to show the effect of matter clumpiness, we simulate the Galactic Halo by simply considering a uniform over-density. A complete treatment of a clumpy universe will be reported elsewhere. We also estimate the effect of very slow decay of DM on the equation of state of the Universe and on the baryon and lepton asymmetries.

In this section we describe the decay model of UHDM and interactions which are included in the simulation.

\subsection{Decay Model}

Theoretical predictions for mass and lifetime of UH particles cover a large range of values $m_{U H}=10^{22}-10^{26} \mathrm{eV}$ and $\tau_{U H}=10^{7}-10^{20} \mathrm{yr}$ [3] [5] [64]. Nevertheless, at the end of inflation it is more difficult to produce the highest range of the masses and special types of inflationary models [65] are needed. For UH particles make a substantial part of the Dark Matter today, their lifetime must be at least comparable to the age of the Universe. In this work we perform the simulation for $m_{U H}=10^{22} \mathrm{eV}$ and $m_{U H}=10^{24} \mathrm{eV}$. For lifetime, $\tau_{U H}=5 \tau_{0}$ and $\tau_{U H}=50 \tau_{0}$, where $\tau_{0}$ is the age of the Universe, are studied. These values are smaller than what have been used by other groups [58] 600]. We show below that taking into account a realistic model for energy dissipation of remnants, even these relatively short lifetime can not explain the flux of UHECRs in a homogeneous universe. For some halo and IR background models, these lifetimes or slightly larger ones are compatible with observations.

The decay modes of UHDM are very model dependent. It is very likely that they don't decay directly to known particles and their decay has a number of intermediate unstable states that decay in their turn. It is also very probable that remnants include stable WIMPs which are not easily observable. To study the maximal effects of the decay on high energy backgrounds, we assume that at the end, the whole decayed energy goes to stable visible particles. 
Most of WIMPZILLA models consider them to be neutral bosons. Due to lack of precise information about their decay, we assume that it looks like the decay of $Z^{\circ}$. Theoretical and experimental arguments show that leptonic and hadronic decay channels of $Z^{\circ}$ have a branching ratio of $\sim 1 / 3-2 / 3$ [66]. As hadronic channel is dominant, here we only consider this mode. It maximizes the flux of nucleons which at present are the dominant observable at ultra high energies.

To mimic the softening of energy spectrum due to multiple decay level, we assume that decay is similar to hadronization of a pair of gluon jets. Experimental data [67] as well as MLLA (Modified Leading Logarithm Approximation) [68], LPHD (Local Parton-Hadron Duality) ( [69] and references therein) and string hadronization model [70] predict a softer spectrum with higher multiplicity for gluon jets than for quarks.

We use PYTHIA program [63] for jet hadronization. This program, like many other available ones, can not properly simulate ultra high energy events, not only because we don't know the exact physics at $10^{16} \mathrm{GeV}$ scale, but also because of programming limits. For this reason, we had to extrapolate simulation results for $E_{C M} \leqslant 10^{20} \mathrm{eV}$ up to $E_{C M}=10^{24} \mathrm{eV}$. Fig.1] shows as an example, proton and photon multiplicity in hadronization of a pair of gluon jets. At middle energies, the multiplicity per $\log (E)$ is roughly constant. The same behavior exists for other species. This is a known shortcoming of present fragmentation simulations [71] (See also Appendix 1) and makes spectrum harder at middle energies. As we would like to study the maximal flux of UHECRs and their effects on high energy backgrounds, this problem can not change our conclusions.

In the simulation, all particles except $e^{ \pm}, p^{ \pm}, \nu, \bar{\nu}$ and $\gamma$ decay. We neglect neutrinos mass and for simplicity we assume only one family of neutrinos i.e. $\nu_{e}$.

Contribution of the stable species in the total multiplicity and the total decay energy is summarized in Table 1. For all species, more than $99 \%$ of the total energy belongs to the particles with energies higher than $10^{20} \mathrm{eV}$ and $10^{18} \mathrm{eV}$ respectively for two masses considered here. Apparently the mass of UHDM has little effect on the composition of remnants. However, one has to admit an uncertainty about this conclusion which is a direct consequence of the uncertain behavior of the multiplicity spectrum as mentioned above and in the Appendix 1.

\section{$2.2 \quad$ Interactions}

We have included roughly all relevant interactions between remnants (except $\nu-\nu$ and $\bar{\nu}-\bar{\nu}$ elastic scattering) to the simulation either analytically or by using the results of PYTHIA Monte 
Table 1: Energy and multiplicity contribution in remnants of WIMPZILLA.

\begin{tabular}{|c|c|c|c|c|}
\hline & \multicolumn{2}{|c|}{$M_{d m}=10^{24} \mathrm{eV}$} & \multicolumn{2}{c|}{$M_{d m}=10^{22} \mathrm{eV}$} \\
\hline Part. & Ener. \% & Multi. \% & Ener. \% & Multi. \% \\
\hline$e^{ \pm}$ & $6.7 \times 2$ & $9.7 \times 2$ & $6.7 \times 2$ & $9.8 \times 2$ \\
$p^{ \pm}$ & $11.8 \times 2$ & $1.4 \times 2$ & $11.9 \times 2$ & $1.4 \times 2$ \\
$\nu \& \bar{\nu}$ & $18.4 \times 2$ & $28.3 \times 2$ & $18.1 \times 2$ & $28.3 \times 2$ \\
$\gamma$ & 26.2 & 21 & 26.6 & 21 \\
\hline
\end{tabular}

Carlo. Previous works [57] [58] [59] [60] 62] either don't consider the energy dissipation or take into account only the first order perturbative interactions (except for $p-\gamma$ where a fitting is used for $N-\gamma \rightarrow N-\pi$ cross-section). An early version of the present simulation [61] studied only energy dissipation of UHECRs using PYTHIA without considering low energy processes and interactions of secondary particles. It found a higher lifetime for UHECRs than present work.

The main reason for considering only first order interactions is that it is usually assumed that interaction with $\mathrm{CMB}$ is dominated by the minimal process i.e $N-\gamma \rightarrow N-\pi$. However, the CMB spectrum even on its peak spans over a wide range of energies where radiation correction and hadronization become important. For instance, at $E_{C M}=4 G e V$ the mean multiplicity is $\sim 15$ in place of 5 (after pion decay) in the minimal interaction. Moreover, in the galactic medium, the IR and visible radiations are comparable with CMB and play an important role in the energy dissipation of protons of $E \sim 10^{18}-10^{19} \mathrm{eV}$. In extragalactic medium, the number density of background high energy photons with $(E>1 \mathrm{eV})$ is larger than visible and near IR. Another factor which accelerates energy dissipation of protons in the interaction with high energy photons is energy loss of leading proton in $p-\gamma$ interaction. It increases with energy (See Fig.2) and results a higher dissipation rate.

PYTHIA can not simulate processes with invariant CM energies $E_{C M} \equiv \sqrt{s}, E_{C M}<2 G e V$ to $E_{C M}<4 G e V$ (the lower limit depends on the interaction). Consequently, for smaller energies we have included only perturbative, first order interactions using analytical expressions. Table 3 summarizes processes which are included in the simulation, the energy range of analytical and/or Monte Carlo calculation of the cross-sections, and the cuts used for removing singularities at small energies or angles in the case of analytical calculation (results depend somehow on these cuts specially in the case of moderate energy and angular resolution of our program). For energy ranges that PYTHIA has been used, in general we use default value of various parameters of the program as defined in PYTHIA manual. A number of parameters have been changed for all 
Table 2: PYTHIA Parameters

\begin{tabular}{|c|l|}
\hline Selected processes & $\begin{array}{l}\text { lept.-lept, lept.-had., unresolved } \gamma, \text { low } p_{t}, \text { diffract. } \\
\text { \& elastic had.-had., Radiative correction }\end{array}$ \\
\hline Status parameters & $2^{\text {ed }}$-order $\alpha_{s}$, continuous $p_{t}$ cutoff. \\
\hline Pt cuts & $E_{C M}^{\text {min }}=1, p_{t}^{\text {min }}=0$ \\
\hline Number of flavors & 6 \\
\hline
\end{tabular}

processes e.g. to make unstable particles like mesons decay etc. They are listed in Table 2. For some interactions, the value of a few other parameters are changed. They are also listed in the Table 3. From now on $s, t$ and $u$ are Mandelstam variables.

At very high energies, $E_{C M}>10^{14} \mathrm{eV}$ for nucleon-nucleon and $E_{C M}>10^{15} \mathrm{eV}$ for other interactions, PYTHIA becomes very slow and the number of rejected events increases rapidly. For these energies we perform a linear extrapolation from lower energies as explained below.

\section{Evolution}

We assume that non-baryonic Dark Matter is totally composed of slowly decaying UH particles. From (2) and (4) below it is evident that width and fraction of UHDM in DM are degenerate and in the evolution equations, decreasing contribution is equivalent to increasing lifetime.

Boltzmann equation for space-time and energy-momentum distribution of a particle $i$ is 74 ] (We use units with $c=\hbar=1$ ):

$$
\begin{aligned}
p^{\mu} \partial_{\mu} f^{(i)}(x, p)-\left(\Gamma_{\nu \rho}^{\mu} p^{\nu} p^{\rho}-e_{i} F_{\nu}^{\mu} p^{\nu}\right) \frac{\partial f^{(i)}}{\partial p^{\mu}}= & -(\mathcal{A}(x, p)+\mathcal{B}(x, p)) f^{(i)}(x, p)+\mathcal{C}(x, p)+ \\
\mathcal{D}(x, p)+\mathcal{E}(x, p) . & \\
\mathcal{A}(x, p)= & \Gamma_{i} m_{i} . \\
\mathcal{B}(x, p)= & \sum_{j} \frac{1}{(2 \pi)^{3} g_{i}} \int d \bar{p}_{j} f^{(j)}\left(x, p_{j}\right) A(s) \sigma_{i j}(s) . \\
\mathcal{C}(x, p)= & \sum_{j} \Gamma_{j} m_{j} \frac{1}{(2 \pi)^{3} g_{i}} \int d \bar{p}_{j} f^{(j)}\left(x, p_{j}\right) \frac{d \mathcal{M}^{(i)}}{d \bar{p}} . \\
\mathcal{D}(x, p) & =\sum_{j, k} \frac{1}{(2 \pi)^{6} g_{i}} \int d \bar{p}_{j} d \bar{p}_{k} f^{(j)}\left(x, p_{j}\right) f^{(k)}\left(x, p_{k}\right) A(s) \frac{d \sigma_{j+k \rightarrow i+\ldots}}{d \bar{p}} .
\end{aligned}
$$


$x$ and $p$ are coordinate and momentum 4-vectors; $f^{(i)}(x, p)$ is the distribution of species $i ; m_{i}$, $e_{i}$ and $\Gamma_{i}$, are its mass, electric charge and width $=1 / \tau_{i}, \tau_{i}$ is the lifetime; $\sigma_{i j}$ is the total interaction cross-section of species $i$ and species $j$ at a fixed $s ; \frac{d \sigma_{j+k \rightarrow i+\ldots}}{d \bar{p}}=\frac{(2 \pi)^{3} E d \sigma}{g_{i} p^{2} d p d \Omega}$ is the Lorantz invariant differential cross-section of production of $i$ in the interaction of $j$ and $k ; g_{i}$ is the number of internal degrees of freedom (e.g. spin, color); $d \bar{p}=\frac{d^{3} p}{E}$. We treat interactions classically, i.e. we consider only two-body interactions and we neglect the interference between outgoing particles. It is a good approximation when the plasma is not degenerate. It is assumed that cross-sections include summation over internal degrees of freedom like spin; $\frac{d \mathcal{M}^{(i)} j}{d \bar{p}}$ is the differential multiplicity of species $i$ in the decay of $j ; \Gamma_{\nu \rho}^{\mu}$ is the connection; $F_{\nu}^{\mu}$ an external electromagnetic field; and finally $\mathcal{E}(x, p)$ presents all other external sources. $A(s)$ is a kinematic factor 75 :

$$
A\left(p_{i}, p_{j}\right)=\left(\left(p_{i} \cdot p_{j}\right)^{2}-m_{i}^{2} m_{j}^{2}\right)^{\frac{1}{2}}=\frac{1}{2}\left(\left(s-m_{i}^{2}-m_{j}^{2}\right)^{2}-4 m_{i}^{2} m_{j}^{2}\right)^{\frac{1}{2}} .
$$

The quantity $A \sigma$ presents the probability of an interaction.

In a homogeneous universe $f(x, p)=f(t,|p|)$ and in (1) the term corresponding to interaction with external electromagnetic field is zero. Therefore, to have a consistent formalism for evolution of distribution of all species, we don't include the synchrotron radiation of high energy electrons in a magnetic field.

We only consider the evolution of stable particles and slowly decaying UHDM. The term (2) concerns only UHDM. In (函), the only non-zero term in the sum is the decay of UHDM. We assume that stable species don't have any interaction with UHDM and corresponding interaction integrals in (3) and (5) are zero. Due to the very large mass of UHDM, its momentum is negligible and we can assume that in comoving frame it is at rest. This permits to use its number density $n_{d m}$ which is more convenient for numerical calculation.

In a homogeneous universe the metric in comoving frame is:

$$
d s^{2}=d t^{2}-a^{2}(t) \delta_{i j} d x^{i} d x^{j}
$$

and with respect to local Lorantz frame (11) to (5) take the following form (in the following the species index indicates one of the stable species):

$$
\begin{aligned}
\frac{\partial f^{(i)}(t, p)}{\partial t}-\frac{\dot{a}}{a} p \frac{\partial f^{(i)}}{\partial p} & =\frac{1}{E}\left(-\mathcal{B}(t, p) f^{(i)}(t, p)+\mathcal{C}(t, p)+\mathcal{D}(t, p)\right) . \\
\mathcal{B}(t, p) & =\sum_{j} \frac{1}{(2 \pi)^{2} g_{i}} \int d p_{j} \frac{p_{j}^{2}}{E_{j}} f^{(j)}\left(t, p_{j}\right) \int d\left(\cos \theta_{i j}\right) A(s) \sigma_{i j}(s) .
\end{aligned}
$$




$$
\begin{aligned}
\mathcal{C}(t, p)= & \frac{E}{4 \pi g_{i} p^{2}} \Gamma_{d m} n_{d m} \frac{d \mathcal{M}^{(i)}}{d p} \\
\mathcal{D}(t, p)= & \sum_{j, k} \frac{1}{(2 \pi)^{5} g_{i}} \int d p_{j} d p_{k} \frac{p_{j}^{2}}{E_{j}} \frac{p_{k}^{2}}{E_{k}} f^{(j)}\left(t, p_{j}\right) f^{(k)}\left(t, p_{k}\right) \int d\left(\cos \theta_{j k}\right) d\left(\cos \theta_{j i}\right) \\
& d \phi_{i} A(s) \frac{d \sigma_{j+k \rightarrow i+\ldots}}{d \bar{p}} . \\
\frac{d n_{d m}}{d t}+\frac{3 \dot{a}}{a} n_{d m}= & -\Gamma_{d m} n_{d m} .
\end{aligned}
$$

In (9) and (11) $s$ depends on the angle between species $j$ and $k$, and $j$ and $i$. Consequently, it is not possible to use cross-sections integrated over angular variables.

Evolution of $a(t)$ is ruled by Einstein equation:

$$
\begin{aligned}
\frac{\dot{a}^{2}}{a^{2}} & =\frac{8 \pi G}{3} T_{00}+\frac{\Lambda}{3} . \\
T^{00}(t) & =\sum_{i} \frac{g_{i}}{2 \pi^{2}} \int d p p^{2} E f^{(i)}(t, p) .
\end{aligned}
$$

In a homogeneous cosmology, $T^{00}$ in Local Lorantz frame is the same as comoving frame and $T_{\text {comov }}^{i i}=a^{-2} T_{\text {Loc. Lor. }}^{i i}$.

Equations (8) and (13) determine the cosmological evolution of species. Due to interaction terms, even in a homogeneous universe, these equations are non-linear and coupled. It is not therefore possible to solve them analytically. Evolution equation for DM can be solved analytically for a short period of time. For other species if in (8) we consider absorption and production integrals as $t$ and $p$ dependent coefficients of a linear partial differential equation, (8) can be solved analytically [76]. Giving the value of $f^{(i)}(t, p), n_{d m}(t)$ and $a(t)$, at time $t$, we can then determine $a(t+\Delta t), n_{d m}(t+\Delta t), \mathcal{B}(t, p), \mathcal{C}(t, p)$ and $\mathcal{D}(t, p)$ for a short time interval $\Delta t . f^{(i)}(t+\Delta t, p)$ would be obtain from solution of partial differential equation (8) using difference method. The solution of metric and distributions in one step of numerical calculation are the followings:

$$
\begin{aligned}
a(t+\Delta t) & =a(t) \exp \left(\Delta t\left(\frac{8 \pi G}{3} T_{00}+\frac{\Lambda}{3}\right)^{\frac{1}{2}}\right) . \\
n_{d m}(t+\Delta t) & =n_{d m}(t) \frac{a^{3}\left(t_{0}\right)}{a^{3}(t)} \exp \left(-\frac{t-t_{0}}{\tau}\right) . \\
f^{(i)}(t+\Delta t, p) & =\left(f^{(i)}\left(t, p^{\prime}\right)+\Delta t\left(\mathcal{C}\left(t, p^{\prime}\right)+\mathcal{D}\left(t, p^{\prime}\right)\right)\right) \exp \left(-\mathcal{B}\left(t, p^{\prime}\right) \Delta t\right) \\
p^{\prime} & =\frac{a(t+\Delta t) p}{a(t)} .
\end{aligned}
$$

This prescription is more precise than a pure numerical calculation using e.g. difference method. 


\section{Numerical Simulation}

What makes numerical calculation of (15) to (17) difficult is the extension on roughly 34 orders of magnitude of energy from $10^{-9} \mathrm{eV}$ (radio background) to $10^{24} \mathrm{eV}$ (mass of UHDM) (from now on we call this energy range $\mathcal{R}_{E}$ ). Physical processes in this vast energy range have varieties of behavior, resonances, etc. Moreover, species have distributions which are orders of magnitude different from each others. In other term, these equations are very stiff. Semi-analytic method explained above helps to increase the precision of the numerical calculation. However, the 5dimensional integration in (11) is extremely time and memory consuming and it is impossible to determine it with the same precision.

In the following we describe in detail the numerical calculation of (15) to (17), as well as cosmological model, initial conditions and backgrounds which have been used.

\subsection{Multiplicity and Cross-section}

For processes simulated by PYTHIA, we need to calculate total and differential cross-sections (see (9) and (11)). The former is given by the program itself. To determine the latter, we divide $\mathcal{R}_{E}$ to logarithmic bins (one per order of magnitude) and classify particles according to their momentum. The angular distribution of produced particles with respect to the axis of incoming particles in CM also is divided linearly to 90 bins. At a given $s$, the cross section in each bin $\Delta \sigma_{i j}=\sigma_{t o t} N_{i j} / N . N_{i j}$ is the number of particles of a given species in the bin $i j . N$ is the total number of simulated events.

The same procedure is used for determination of $\frac{d \mathcal{M}}{d p}$. In this case it is not necessary to consider the angular distribution because in the rest frame of WIMPZILLA the decay has a spherical symmetry.

Because PYTHIA can not cover the totality of the energy range, for high energy bins we use a linear extrapolation in $\log p$. The contribution of these energies i.e. $E_{C M} \gtrsim 10^{6} \mathrm{GeV}$ on the evolution of species is nevertheless small because the density of concerning particles is very low. The reason for adding them is not to have an artificial cut in the calculation.

As mentioned above, for most processes including $p-\gamma$, PYTHIA can not simulate the interaction with $E_{C M}<4 G e V \cdot p-\gamma$ is the most important process for the energy dissipation of protons specially in this uncovered energy range where interaction with CMB photon is concentrated. In these energies we use directly the total cross-section obtained from experience [66]. For differential cross-section, we extrapolate angular distribution from higher energies and normalize it to the exact total cross-section. 


\subsection{Evolution Equations}

In (9) and (11), the integrals over angular degrees of freedom can be separated from energy integrals and they don't depend on any cosmological or DM parameter. It is therefore very convenient to calculate them separately.

We divide each $180^{\circ}$ interval to 9 bins and use trapezoid method for integration. For the single integral in (9) a better resolution with 90 bins has been used. However, our tests show that even the moderate resolution of 9 bins gives, up to a few percents, the same results as the more precise integration. This is a reassuring results and means that the triple integrals in production term also must be enough correct even with a low resolution.

Calculation of production term for first-order interactions is more complicate. They are processes of type 2 part. $\rightarrow 2$ part. In the CM frame where all cross-sections are determined, analytically or numerically, the incoming and outgoing particles have the same momentum. This is equivalent to having a Delta function in the integrand. The numerical realization of this function specially with a moderate resolution is very difficult. Consequently, one has to analytically absorb this function into integrand. Details of the calculation can be found in Appendix 2.

Numerical solution of evolution equation itself needs much better energy resolution due to stiffness of distributions. The resolution must be at least comparable to smallest quantities. Our tests show that a division to 680 logarithmic bins of the energy range $\mathcal{R}_{E}$ (i.e 20 bins per one order of magnitude) gives an acceptable compromise between precision and calculation time.

We divide the interval $z=\left[z_{\text {dec }}-0.001\right]$ to 30 logarithmic bins and the last step is from $z=0.001$ to $z=0$. The program is written in $C^{++}$language and is highly modulable. It can be requested from the author.

To test the precision of our numerical calculation, we have run the program without interaction terms. The error on total $T^{00}$ is $0.7 \%$ and on non-baryonic DM is practically zero. For other species it is $5.5 \%$ to $7.5 \%$. Including interaction terms but not the decay of DM gives the same answer. This test is crucial for correct simulation of thermal equilibrium of the Universe.

\subsection{Cosmology Model and Initial Conditions}

We consider a flat universe with present value of parameters as the followings: $\Omega_{M}=0.3$, $\Omega_{\Lambda}=0.7, h=H_{0} / 100 \mathrm{~km} \mathrm{sec}^{-1} M p c^{-1}=0.7$ and $\Omega_{b}=0.02 h^{-2}$.

We fix the decoupling redshift at $z_{d e c}=1100$. The distribution of species at that time was

thermal with a temperature $T_{d e c}=T_{c m b}\left(z_{d e c}+1\right)=0.26 \mathrm{eV}, T_{c m b}=2.728 \mathrm{~K}$ [77 for $e^{ \pm}, p^{ \pm}$and $\gamma$ and $\frac{4}{11} T_{\text {dec }}$ for $\nu$ and $\bar{\nu}$. 
In the same way, one can determine the temperature of Dark Matter 9 at decoupling [78:

$$
T_{d m}\left(z_{d e c}\right)=\frac{g_{* s}^{d e c} \frac{2}{3} T_{d e c}^{2}}{g_{* s}^{d m} \frac{2}{3}} T_{d m-d e c}
$$

$T_{d m-d e c}$ is the decoupling temperature of the Dark Matter. If we assume that $T_{d m-d e c} \sim 10^{16} \mathrm{eV}$, $T_{d m}\left(z_{d e c}\right)<10^{-18} \mathrm{eV}$. Therefore, our approximation $T_{d m}=0$ is quite justified.

We know the density of species at present. Their initial value at decoupling depends on the equation of state of the Universe. However, it is exactly what we want to calculate! Consequently, we have to determine their value at decoupling approximately by neglecting the effect of UHDM decay. As the lifetime of WIMPZILLA is assumed to be much longer than the age of the Universe, the present value of densities after evolution must stay very close to our initial assumption.

We define the initial densities as the followings:

$$
\begin{aligned}
n_{\gamma}=n_{C O B E}\left(z_{d e c}+1\right)^{3} & n_{\nu}=n_{\bar{\nu}}=3 \times \frac{4}{11} \times n_{\gamma} \\
n_{p}=\frac{\Omega_{b} \rho_{c}}{m_{p}}\left(z_{d e c}+1\right)^{3} & n_{e^{-}}=n_{p} \quad n_{\bar{p}}=n_{e^{+}}=0 \\
n_{d m}=\frac{\rho_{c}\left(\Omega_{M}-\Omega_{h o t}-\Omega_{b}\left(1+\frac{m_{e}}{m_{p}}\right)\right)}{m_{d m}}\left(z_{d e c}+1\right)^{3} & \Omega_{h o t}=\frac{\pi^{2}}{30} g_{*} T_{c m b}^{4} .
\end{aligned}
$$

Knowing initial density and temperature, other quantities like chemical potential and distributions can be determined $[78]$. We assume that the age of the Universe $\tau_{0}=14.8$ Gyr. This quantity also depends on the equation of state and we use it only for fixing the lifetime of WIMPZILLA.

\subsection{Backgrounds}

Apart from $\mathrm{CMB}$ and relic neutrinos which are included in the initial conditions, we don't include any other background to high redshift distributions. For $z \leq 3$, we add near-IR to UV emissivity of stars [79] [80] to equation (17). Far-IR which is very important for energy dissipation of UHECRs, is not added because there is very little information about its evolution with redshift. High energy backgrounds are not added because we want to be able to distinguish the contribution of remnants from other sources.

Adding backgrounds by "hand" evidently violates the energy conservation of the model, but there is not any other simple alternative method. Moreover, the violation is very small and comparable to numerical errors.

\footnotetext{
${ }^{2}$ One should consider $T_{d m}$ as an estimation of kinetic energy scale rather than a real temperature because if ultra heavy particles exist, they could never be thermalized.
} 
Here a comment is in order: why have we included star backgrounds and not the synchrotron radiation ? Star background is considered as an external source. By contrast, synchrotron radiation concerns high energy electrons which are involved in the evolution. In (1), the elimination of interaction with an external field in a homogeneous universe means that the probability for production and absorption of synchrotron photons is the same. It is not therefore possible to consider their production without their absorption i.e. interaction of electrons with external magnetic field.

\section{$5 \quad$ Results}

Figure 3 shows the energy flux of high energy protons and photons in a homogeneous universe. Fig. 1 shows the same quantity for all species. The GZK cutoff is very transparent. With our

background model it begins at $E \approx 10^{18.2} \mathrm{eV}$ for protons due to $p-\gamma$ interaction (see optical depth in Fig. [5) and at $E \sim 10^{13} \mathrm{eV}$ for photons due to $e^{ \pm}$production. For purely kinematic reasons, proton cutoff is much shallower than photon one. The resonance of $p-\gamma$ interaction is very close to $p$ rest mass where $A(s)$ in (3) is very small. Moreover, division of (8) by $E$ reduces the effect of absorption on the distribution.

According to Fig.3, even the shortest lifetime we have considered, can not explain the observed flux of protons (in contrast to 62 that assumes a 2-particle decay mode). The same figure shows also the flux without energy dissipation. It is compatible with Ref. [60] which assumes a hadronic decay but does not consider the energy dissipation of secondary particles. In this latter case, the lifetime must be $\sim 4-6$ orders of magnitude larger than the age of the Universe. On the one hand, this result proves the rôle of a realistic model of energy dissipation in the estimation of mass and lifetime of UHDM. On the other hand, it shows the importance of clumping of Dark Matter, i.e. most of observed UHECRs must come from nearby sources. This conclusion is independent of the source of UHECRs. A similar conclusion has been obtained in [58 by fitting the expected flux from extragalactic sources and from the Halo on the data. However they don't consider the dissipation. In the next section we show that even in a halo, the dissipation of photons energy is significant.

In the case of decaying UHDM hypothesis, the most important source is the Galactic Halo. Before trying to make a simple model of halo in the next section, we discuss some of other conclusions one can make from this study.

From Fig. 3 and by taking into account the fact that $p-\gamma$ cross-section is $\sim 10^{4}$ times smaller than $p-p$, with present statistics of UHECRs, less than one photon shower could be observed. The comparison of EGRET data for $10^{8} \mathrm{eV}<E<10^{11} \mathrm{eV}$ with our calculation shows that it 
is compatible with a short lifetime UHDM. Future observations of GLAST at $E>10^{11} \mathrm{eV}$ is crucial for understanding the source of UHECRs because one expects an additional contribution from synchrotron radiation of ultra high energy electrons at $E \sim 10^{12}-10^{14} \mathrm{eV}$ [59] [62].

Fig. . illustrates the total and partial optical depth, i.e. $\mathcal{B}(t, p)$ in equation (9) at $z=0$. It shows that for protons with $E \sim 10^{20} \mathrm{eV}$, even a source at $\sim 20 \mathrm{Mpc}$ must be very strong to be able to provide the observed flux because its flux is reduced by 11 orders of magnitude! The optical depth of protons must be even larger than what we have obtained here because we didn't take into account the far-IR and radio backgrounds. This makes difficulties for the recent suggestion by Ahn E.J., et al. [40] that Virgo cluster can be the only source of UHECRs, even if the magnetic field of Galactic wind is as strong as what is considered in that work. The main challenge is finding a conventional source with enough emissivity of CRs at ultra high energies.

\subsection{CMB Distortion and Entropy Excess}

To see if a relatively short living UHDM can distort CMB, we reduced the spectrum of photons for a decaying DM from the spectrum with a stable DM. Fig.6 shows the result for $z=0$. There is no distortion at least up to 1 to $10^{8}$ parts for $E \lesssim 3 \mathrm{eV}$. However, one should note that this conclusion depends somehow on the cross-section cuts at low energies. Nevertheless, giving the fact that CMB flux at its maximum is $\sim 10^{20}$ times larger than the rest of the spectrum, it seems very unlikely that it can be disturbed, otherwise SZ effect due to stars had to be observed everywhere. The distortion of CMB anisotropy will be studied elsewhere.

We have also examined the entropy excess separately for each species. There is no entropy enhancement except for $e^{+}$and $p^{-}$which in our model are absent from the initial conditions. Comparing to other species, their contribution is very small and negligible.

\subsection{Baryon and Lepton Asymmetry Generation}

It has been suggested [81] [22] that the decay of UHDM may be able to generate additional baryon and lepton asymmetry. At GUT scale, i.e. the mass scale of WIMPZILLA, we expect such processes and the fact that a late time decay is out of thermal equilibrium and satisfies Sakharov conditions for baryogenesis [78] make growing asymmetry plausible.

The rate of baryonic (or leptonic) number production by decay of UHDM in comoving frame can be expressed as:

$$
\frac{d\left(n_{b}-n_{\bar{b}}\right)}{d t}+3 \frac{\dot{a}(t)}{a(t)}\left(n_{b}-n_{\bar{b}}\right)=\frac{\varepsilon n_{d m}}{\tau} .
$$


$\varepsilon$ is the total baryon number violation per decay. The solution of this equation is:

$$
\begin{aligned}
\Delta\left(n_{b}-n_{\bar{b}}\right) & =\varepsilon n_{d m}\left(t_{0}\right)\left(1-\exp \left(-\frac{t-t_{0}}{\tau}\right)\right) \frac{\left(1+z_{0}\right)^{3}}{(1+z)^{3}} \\
\Delta B & \equiv \frac{\Delta\left(n_{b}-n_{\bar{b}}\right)}{2 g_{*} n_{\gamma}}=\frac{\varepsilon n_{d m}\left(t_{0}\right)}{2 g_{*} n_{\gamma}\left(t_{0}\right)}\left(1-\exp \left(-\frac{t-t_{0}}{\tau}\right)\right) \frac{(1+z)}{\left(1+z_{0}\right)} .
\end{aligned}
$$

If $t_{0}=t_{d e c}, \frac{n_{d m}\left(t_{0}\right)}{n_{\gamma}\left(t_{0}\right)} \sim 10^{-22}$ (for $m_{d m}=10^{24} \mathrm{eV}$ ). Therefore $\Delta B \sim 10^{-22} \varepsilon$ at $z=0$. As $\varepsilon$ can not be larger than total multiplicity, $\sim 1000, \Delta B \lesssim 10^{-19}$, i.e. much smaller than primordial value $\sim 10^{-10}$.

We tested this argument by assuming $\varepsilon=0.1$ at all energies, i.e. $\varepsilon_{\text {tot }}=0.1 \mathcal{M}_{\text {tot }}$. Evidently $n_{\bar{p}}$ is smaller, but the change of $n_{p}$ is too small to be measured. The same is true for number density of leptons, but energy density of leptons with respect to anti-leptons increases by an amount comparable to $\varepsilon$.

\subsection{Equation of State of the Universe}

Decay of UHDM gradually changes part of CDM to HDM and thus changes the equation of state of the Universe. Fig. 7 shows the variation of equation of state. For $\tau \sim 50 \tau_{0}$ or larger, it would be too small to be measurable. For smaller $\tau$, DM decay plays the rôle of a running cosmological constant. A complete study of this issue and comparison with data is under preparation.

\section{Halo}

To see the effect of clumping of a decaying UHDM on the flux of UHECRs, here we try to make a very simple model. A complete treatment of halos will be reported elsewhere.

We consider a halo as a uniform over-density with a limited size at $z=0$. This is simulated by following the decay of UHDM and evolution of remnants for a time comparable to the propagation time in the halo. Evolution equation and energy binning is taken to be the same as in the homogeneous universe case with $a(t)=c t e$. Because we want to study the propagation of remnants in a volume comparable to the Galactic Halo, we consider time steps equivalent to $10 k p c$. Our tests show that after a few steps $(\sim 7)$, the accumulation rate of ultra high energy particles becomes very slow. We consider two cases. In the first case we simply evolve distributions for a number of steps (up to 30). In the second case, after some steps, we stop the decay of the Dark Matter to simulate an inner halo of MACHOs. Then, the evolution is continued for more 5 steps (i.e. 50kpc) 84 to simulate propagation through MACHOs. Evidently this model is very approximative. We use it only to make a crude estimate of production and 
absorption of UHECRs.

\subsection{Initial Conditions and Galactic Backgrounds}

Galactic baryonic matter is taken to have a thermal distribution with $T_{b}=10^{4} \mathrm{~K}$. We assume that baryonic over-density is biased with respect to DM i.e. the fraction of baryons to DM is larger than its mean value in the Universe. With these assumptions the initial number densities can be expressed as:

$$
\begin{array}{r}
n_{p}=\frac{b \delta \rho_{c}}{m_{p}} \quad n_{e^{-}}=n_{p} \\
n_{\bar{p}}=n_{e^{+}}=0 \\
n_{d m}=\frac{(1-b) \delta \rho_{c}}{m_{d m}}
\end{array}
$$

$b$ is the fraction of baryons in the halo. In the following $b=0.3$, i.e. $\sim 2$ times primordial value [85] in the cosmological model explained above. $\delta$ is the mean over-density of the Halo. Inspired by universal halo density distribution of NFW [86], we consider a halo with characteristic radius (i.e. virial radius) $r_{200}=0.12 \mathrm{Mpc}$. According to $\mathrm{NFW}$ distribution and by definition this means $\delta=200$. These parameters defines a halo of mass $M_{H}=6 \times 10^{12} M_{\odot}$.

Neutrino density is assumed to be the same as relic at $z=0$. For photons, in addition to CMB, we consider a galactic background as the following:

Galactic IR and visible backgrounds are not very well known. We use the results of the model developed by DIRBE group for detection of extragalactic component of the IRB [87. We consider the observed value of IRB after elimination of Inter-Planetary Dust (IDP) contribution as the galactic background. It is just an estimation of average galactic IRB. It is not clear if we can extend the local value of IRB to whole galaxy or take it as a representative average. For this reason we also increase it 10 times (probably an extremely high value) to see the effect on the energy dissipation of UHECRs (see below for conclusions). Our simulation does not include radio background.

For soft and hard X-Ray galactic backgrounds, we use the model developed for extraction of extragalactic component from ROSAT and ASCA observations 88. It considers GXB as two thermal components, a soft component with $T_{s x}=70 \mathrm{eV}$ from Local Bubble, and a hard component with $T_{h x}=145 \mathrm{eV}$ from hot gas, probably in the Halo. We add also the extragalactic component for $0.25 \mathrm{keV}<E<10 \mathrm{keV}$. 


\section{$7 \quad$ Results}

Fig. 8 shows the distribution of high energy protons and photons for a uniform halo and for a halo that its inner part is composed of MACHOs. Only the result for $m_{d m}=10^{24} \mathrm{eV}$ with $\tau=5 \tau_{0}$ and $\tau=50 \tau_{0}$ is shown. Because of importance of the spectrum close to observed trough in the interpretation of the results, Table 1 summarizes the numerical value of simulated and observed spectrum.

For photons the trough of GZK cutoff is shallower than in a homogeneous universe and for protons it is practically absent. Consequently, the calculated flux at $E \sim 10^{19.5} \mathrm{eV}$ is somehow higher than observation. However, at higher energies simulation results specially one with a MACHO halo is in the $1-\sigma$ error range of the observations.

The simplest explanation of having a smaller observed flux close to the minimum of the spectrum can be the need for increasing the lifetime somehow (but not by many orders of magnitude as suggested by previous works [60] [62]). However, the lack of a minimum in the simulated spectrum ${ }^{3}$ means that in some way our simulation is not exact. It can be due to a too simple halo model. Other possibilities are a different (probably harder) decay spectrum and/or energy loss in the Galactic magnetic field. If these suggestions are true, fluxes will be smaller and there is no need for increasing the lifetime. A more complete simulation of the Halo and magnetic field and a better understanding of non-baryonic DM distribution is necessary for making any definitive conclusion.

The minimum in the spectrum can also be interpreted as a wider distribution of sources. In this case, it is hardly probable that sources responsible for CRs at lower energies can explain this behavior because even if the spectrum of these sources can be extrapolated with the same slop to higher energies, it can not explain the rising slop of the spectrum.

As the IR background is crucial for energy dissipation of UHECRs, we have also increased it to 10 times of the DIRBE model to see the effect. Proton flux at high energies slightly decreases, but it can not explain the observed minimum. We have also tested the distortion of the CMB by remnants as described for a homogeneous universe. There is no distortion up to at least 1 to $10^{8}$ for $E \lesssim 10 \mathrm{eV}$.

Summarizing this section, it seems that a decaying UHDM with a lifetime as short as $\tau \sim$ $10-100 \tau_{0}$ is not ruled out by present observations.

\footnotetext{
${ }^{3}$ In fact optical depth has a maximum at $E \approx 3.3 \times 10^{19} \mathrm{eV}$.
} 


\section{Conclusions}

The main purpose of this work was showing the importance of a realistic physical model for finding the answer to the mystery of UHECRs and introducing readers to the program that has been developed for achieving this goal.

We showed that $\mathcal{R} \equiv \xi \tau_{0} / \tau \sim 0.1-0.01$ where $\xi$ is the contribution of UHDM in the Dark Matter. This value is larger (or equivalently the lifetime is shorter for the same contribution) than what has been suggested in previous works which don't consider the dissipation [58] [60]. If a more realistic halo model confirms this conclusion, the cosmological implication of a UHDM can be important.

We have studied a special decay mode. Any other mode that produces invisible WIMPs or leptonic or semi-leptonic modes decreases the lifetime of UHDM. If more nucleon are produced, the lifetime must be longer but it seems less probable than other cases.

We showed the reciprocal influence of UHECRs and backgrounds on each others. Consequently, whatever the source of UHECRs, it is extremely important to correlate their observations to the observation of high energy photon and neutrino backgrounds.

This work is the first step to a comprehensive study of the effects of a decaying Dark Matter. Other issues like a realistic model for halos, effects on the determination of cosmological parameters and equation of state and comparison with more data from cosmic rays and high energy backgrounds remain for future works.

Note: Shortly after completion of this work the Lake Baikal Experiment collaboration 90 have published their upper limit on the flux of high energy neutrinos. It is well above what is obtained in our simulation (see Fig.5).

\section{Appendix 1: Fragmentation in MLLA}

The MLLA treats fragmentation as a Markov process. Consequently, the differential multiplicity is proportional to splitting function $P(x)$ (See e.g. [69]):

$$
\frac{d \mathcal{M}\left(x_{E}\right)}{d x_{E}} \propto P(x) \quad x_{E}=\frac{E}{E_{j}} .
$$

For a gluon jet and at $x_{E} \ll 1, P\left(x_{E}\right) \sim \frac{1}{x_{E}}$, and one expects that:

$$
\frac{d \mathcal{M}\left(x_{E}\right)}{d\left(\ln \left(x_{E}\right)\right)} \propto \text { Total Num. of splitting } \propto E_{j}
$$


In PYTHIA however, at high energies $E \gtrsim 100 \mathrm{GeV}$, increasing CM energy just increases the probability of having more high energy fragments which simply escape fragmentation. A consequence of this behavior is the narrowing of the multiplicity distribution (See Fig.9) in contrast to theoretical prediction i.e. KNO [89] scaling for $E_{j} \rightarrow \infty$. This limit is obtained at parton level. However, if LPHD is valid, one expects the same type of behavior at hadron level. Another factor that can explain, at least partially, the deviation from theory is the decay of hadrons in our simulation. It increases total multiplicity more than its statistical variation and makes the distribution narrower, but it can not explain the absence of a tail of large multiplicity events. Even after decay, these events should keep their difference with average. As both MLLA and Monte Carlos fail to reproduce observations in relatively low energies [67] [71], the exact behavior at high energies is not clear.

\section{Appendix 2: Production Integral for $2 \rightarrow 2$ Processes}

Cross-sections are Lorantz invariant. However, to have a unique expression for what are simulated and what are calculated analytically, we determine all of them in their CM.

The triple integral in (11) depends on three momentum variables corresponding to the momentum of two incoming particles and one of the outgoing particles that its evolution is under calculation. In the case of a $2 \rightarrow 2$ process, the amplitude of the momentum of final particles depends only on s. If:

$$
\mathbf{p}=(E, p \cos \phi \sin \theta, p \sin \phi \sin \theta, p \cos \theta)
$$

is the 4-momentum of outgoing particle, and $\mathbf{p}^{\prime}$ is its counterpart in the CM:

$$
\begin{aligned}
\mathbf{p}^{\prime 2} & =\frac{\left(s-m^{2}-m^{\prime 2}\right)^{2}-4 m^{2} m^{\prime 2}}{4 s} . \\
\mathbf{p}^{\prime} & =\Gamma \mathbf{p}
\end{aligned}
$$

$m$ and $m^{\prime}$ are the mass of out-going particles, $\Gamma$ is the boost matrix. The equality of two expressions for $\mathbf{p}^{\prime}$ leads to an equation that can be solved for one of the angular variables in (29). The calculation is tedious but strait forward. With respect to $\phi$, the equation is $4^{\text {th }}$ order but in the case of a homogeneous cosmology where the boost matrix depends only on the relative angle between incoming particles, it depends only on $\tan ^{2}\left(\frac{\phi}{2}\right)$ and is analytically solvable. In this way the integration over $\phi$ in (11) reduces to sum of integrand evaluated at the roots of the equation.

This method provides a general way to deal with this problem and is more convenient than 
doing calculation for each cross-section separately.

Acknowledgement I would like to thanks people of Strasburg Observatory for their kindness.

\section{References}

[1] Chung D., Kolb E. W., Riotto A., hep-ph/9810361.

[2] Chung D., Kolb E. W., Riotto A., Phys. Rev. D 59, 023501 (1999), Kuzmin V. \& Tkachev I., JETPhys. Lett. 68, 271 (1998), Chung D., hep-ph/9809489.

[3] Ellis J., Lopez J.L., Nanopoulos D.V. Phys. Lett. B245375 1990.

[4] Witten E., Nucl. Phys. B 471, 135 (1996).

[5] Benakli K. et al., Phys. Rev. D 59, 047301 (1999).

[6] Giudice G.F. \& Rattazzi R. hep-ph/9801271.

[7] Hamaguchi K., Nomura Y., Yanagida T., Phys. Rev. D 58, 103503 (1998), Phys. Rev. D 59, 063507 (1999).

[8] Kofman L., Linde A., Starobinsky A. A. Phys. Rev. Lett. 73, 3195 (1994), Phys. Rev. D 56, 3258 (1997), Khlebnikov, S. \& Tkachev I., Phys. Rev. Lett. 77, 219 (1996), Phys. Rev. Lett. 79, 1607 (1997), Felder G., Kofman L., Linde A., Phys. Rev. D 59, 123523 (1999), Giudice G.F. et al., J. High Ener. Phys. 9908, 014 (1999).

[9] Griest K., Kamionkowski Phys. Rev. Lett. 64, 615 (1990).

[10] Egorov T. A., Proc. Tokyo Workshop on Techniques for the Study of Extremely High Energy Cosmic Rays (ICRR University of Tokyo) p35 (1993).

[11] Bird D.J. et al., ApJ. 424, 491 (1994).

[12] Hayashida N. et al., Phys. Rev. Lett. 73, 3491 (1994), Yoshida S. et al., Astropart. Phys. 3, 105 (1995).

[13] Yoshida Sh. \& Dai H., J. Phys. G: Nucl. Part. Phys. 24, 905 (1998).

[14] Greisen, K., Phys. Rev. Lett. 16, 748 (1966), Zatsepin G.T. \& Kuzmin V.A., JETPhys. Lett. 4, 78 (1966).

[15] Takeda M. et al., astro-ph/9902239. 
[16] Wiebel-Sooth \& Biermann, Landolt-Börnstein vol. VI/3c, Springer Verlag, 37, (1999).

[17] Halzen F. et al., Astropart. Phys. 3, 151 (1995), Boothby K. et al., ApJ. 491, L35 (1997). Cillis \& Sciutto astro-ph/9908002.

[18] Abu-Zayyad, et al., astro-ph/9911144.

[19] Anchordoqui L.A., et al., Phys. Rev. D 59, 094003 (1999),

[20] Epele L.N. \& Roulet E., J. High Ener. Phys. 10, 009 (1998).

[21] Stecker F.W. \& Salamon M.H., ApJ. 512, 20 (1999), Anchordoqui L.A., et al., Phys. Rev. D 60, 103001 (1999), astro-ph/9912081.

[22] Bhattacharjee P. \& Sigl G., astro-ph/9811011.

[23] Blandford R. astro-ph/9906026.

[24] Wandel A., astro-ph/9709133.

[25] Yock, P., in "Black Holes 8 High Energy Astrophysics", Ed. Sato H. \& Sugiyama N., Frontier Science Series No. 23, Universal Academy Press, Tokyo, (1998), Normille, D., Science 284, 734 (1999), Abe F. et al., astro-ph/9911398.

[26] Rachen J.P., Biermann P.L., A.\& A. 272, 161 (1993), Ostrowski astr-ph/9803299.

[27] Halzen F, Vazquez R., Stanev T. \& Vankov H.P., Astropart. Phys. 3, 151 (1995), Halzen F. astro-ph/9704020.

[28] Boldt E. \& Ghosh P., astro-ph/9902342.

[29] Biermann P.L., astro-ph/9501006.

[30] Mannheim K., Protheroe R.J. \& Rachen J.R, astro-ph/9812398.

[31] Miralda-Escudé J., Waxman E., ApJ. 462, L59 (1996), Waxman E., astro-ph/9612061, Totani T., astro-ph/9810206, Astropart. Phys. 11, 451 (1999).

[32] Waxman E., Coppi P., ApJ. 464, L75 (1996).

[33] Achterberg A. et al.astro-ph/9907060.

[34] Harari D. et al., JHE 08, 022 (1999), Farrar G.R., Piran T., astro-ph/9906431.

[35] Sigl G. et al., Astropart. Phys. 10, 141 (1999).

[36] Blasi P., Olinto A.V., Phys. Rev. D 59, 023001 (1999). 
[37] Medina Tanco G.A., de Gouveia Dal Pino E. \& Horvath J.E., Astropart. Phys. 6, 337 (1997), ApJ. 492, 200 (1998), Medina Tanco G.A. astro-ph/9809219, astro-ph/9901053.

[38] Stanev T. et al., Phys. Rev. Lett. 75, 3056 (1995), Uchihori Y. et al., astro-ph/9908139.

[39] Elbert J.W., \& Sommer P., ApJ. 441, 151 (1995), Bird et al., astro-ph/9806096, Hillas M. Nature 395, 15 (1998), Takeda M. et al., Phys. Rev. Lett. 81, 1163 (1998).

[40] Ahn E.J., et al., astro-ph/9911123.

[41] Malkov M.A., ApJ. 511, L53 (1999).

[42] Farrar G.R. \& Biermann P.L., Phys. Rev. Lett. 81, 3579 (1998), Chadwick et al., astro$\mathrm{ph} / 9903346$.

[43] Fargion D., Mele B., astro-ph/9710029, astro-ph/9902024, Weiler T.J., Astropart. Phys. 11, 303 (1999).

[44] Waxman E., astro-ph/9804023, Blanco-Pillado J.J., Vázquez R.A. \& Zas E., astro$\mathrm{ph} / 9902266$.

[45] Dubovsky S.L. \& Tinyakov P.G., Pisma Zh. Eksp. Teor. Fiz 68, 99 (1998), JETPhys. Lett. 68, 107 (1998),

[46] Berezinsky V. \& Mikhailov A.A., Phys. Lett. B 449, 237 (1999)

[47] Hayashida N. et al., Astropart. Phys. 10, 303 (1999), astro-ph/9906056.

[48] Benson A., Smialkowski A. \& Wolfendale A.W. Astropart. Phys. 10, 313 (1999).

[49] Medina Tanco G.A. \& Watson A.A., Astropart. Phys. 12, 25 (1999).

[50] Bhattacharjee P., Hill C.T., Schramm D.N., Phys. Rev. Lett. 69, 567 (1992).

[51] C.T. Hill, Nucl. Phys. B 224, 469 (1983), C.T. Hill, D.N. Schramm \& T.P. Walker, Phys. Rev. D 36, 1007 (1987), Sigl G, Lee S., Schramm D.N., Bhattacharjee P., 1994 (3), Sigl G, Bhattacharjee P., Schramm D.N., astro-ph/9403093, Sigl G., Space Sci. Rev. 75, 375 (1996), Bhattacharjee P., Phys. Rev. Lett. 81, 260 (1998).

[52] Vilenkin A., Shellard E.P.S., "Cosmic Strings and Other Topological Defects", Cambridge University Press, Cambridge (1994).

[53] Albrecht A., Battye R.A., Robinson J., Phys. Rev. Lett. 79, 4736 (1997), Turok N., Pen U.L., Seljak U., Phys. Rev. D 58, 506 (1998), Pen U.L., Seljak U., Turok N., Phys. Rev. 
Lett. 79, 1611 (1997), Albrecht A., Proceedings of the "International Workshop on Particle

Physics and the Early Universe" Ambleside, September 1997, L. Roszkowski Ed.

[54] Contaldi C., Hindmarsh M. \& Magueijo J., Phys. Rev. Lett. 82, 679 (1999), Phys. Rev. Lett. 82, 2034 (1999).

[55] Jedamzik K. Niemeyer J.C., Phys. Rev. Lett. 80, 5481 (1998), Phys. Rev. D 59, 124013 (1999), astro-ph/9901293.

[56] Green A.M., Phys. Rev. D 60, 063516 (1999).

[57] Bhattacharjee P., Phys. Rev. D 40, 3968 (1989), Bhattacharjee P., Hill C.T., Schramm D.N., Phys. Rev. Lett. 69, 567 (1992), Aharonian F.A., Bhattacharjee P., Schramm D.N., Phys. Rev. D 46, 4188 (1992), Protheroe R.J. \& Stanev T., Phys. Rev. Lett. 77, (1996), Sigl G., Lee S. \& Coppi P., astro-ph/9604093, Berezinsky V. \& Vilenkin A., Phys. Rev. Lett. 79, 5202 (1997), , Bhattacharjee P., astro-ph/9803029, Berezinsky V., Blasi P. \& Vilenkin A., astro-ph/9803271.

[58] Berezinsky V., Kachelrie $\beta$ \& Vilenkin A., Phys. Rev. Lett. 79, 4302 (1997).

[59] Lee S., Phys. Rev. D 58, 043004 (1998).

[60] Birkel M.\& Sarkar S., Astropart. Phys. 9, 297 (1998).

[61] Ziaeepour H., Proc. of 2nd Intern. Workshop on the Identification of Dark Matter, Ed. Spooner N. \& Kudryavtsev V., p. 106, World Scientific, 1999 astro-ph/9811312).

[62] Kalashev O.E., Kuzmin V.A. \& Semikoz D.V. astro-ph/9911035.

[63] Sjostrand T., Comp. Phys. Com. 82, 74 (1994).

[64] Han T., Yanagida T. \& Zhang R.J., Phys. Rev. D 58, 095011 (1998), Farnk M, Hamidian H, \& Puolamäki, Phys. Rev. D 60, 095011 (1999).

[65] Felder G, Kofman L \& Linde A., Phys. Rev. D 59, 123523 (1999).

[66] Particle Data Group, Review of Particle Physices, Europ. J. Phys. C 3, 227 (1998).

[67] DELPHI Collab. EPS-HEP99 paper 3-146, OPAL Collab. EPS-HEP99 paper 1-4, Abbiendi G. et al., Europ. J. Phys. C 11, 217 (1999).

[68] Muller A.H., Phys. Rev. D 4, 150 (1971).

[69] Khoze V. A. \& Ochs W., Mod. Phys. A 12, 2949 (1997). 
[70] Andersson et al., Phys. Rep. 97, 31 (1983), Sjöstrand, Nucl. Phys. B 248, 469 (1984), Int. J. Mod. Phys. A3, 751 (1988).

[71] Webber B.R., hep-ph/9912292 and references therein.

[72] Landau L.D., Lifshitz E.M. \& Berestetskii B., Relativistic Quantum Theory, Pergmon Press (1971).

[73] Hannestad S. \& Madsen J., Phys. Rev. D 52, 1764 (1995), Dolgov A.D., Hansen S.H. \& Semikoz D.V., Nucl. Phys. B 503, 426 (1997).

[74] Ehlers J., 1971, in "General Relativity and Cosmology", ed. B.K. Sachs, Academic Press NewYork.

[75] Tauber G.E. \& Weinberg J.W., Phys. Rev. 122, 1342 (1961), Weinberg S., The Quantum Theory of Fields Vol. I, Cambridge University Press, 1996.

[76] See for example Courant R. \& Hilbert B. Methods of Mathematical Physics, John Wiley \& Sons Inc. 1962.

[77] Mather J.C., et al.ApJ. 354, L37 (1990).

[78] Kolb E. \& Turner M., "The Early Universe”, Addison Wesley Publ. Comp. (1990.

[79] Salamon M.H. \& Stecker F.W., astro-ph/9704166.

[80] Valls-Gabaud D. \& Vernet J., to appear in the proceedings of "Chemical Evolution from Zero to High Redshift”, ESO, Garching, Germany, Oct. 1998.

[81] Ziaeepour H., in Proceedings of "From Quantum Fluctuations to Cosmological Structures", Casablanca 1-10 Dec. 1996, Ed. Valls-Gabaud D. et al., ASPC Series vol. 126, p575, Ziaeepour H., in Proceeding of the "Large-Scale Structures: Tracks and Traces", Workshop, Potsdam, 15-20 Sep. (1997), Eds. Müller et al., World Scientific 1998, p. 57.

[82] Sreekumar P., et al., et al., ApJ. 494, 523 (1998).

[83] Chantell M.C., et al., Phys. Rev. Lett. 79, 1805 (1997).

[84] Alcock C., et al., ApJ. 471, 774 (1996), ApJ. 486, 697 (1997).

[85] Jenkins A., et al., ApJ. 499, 21 (1998).

[86] Navarro J., Frenk C. \& White S., ApJ. 462, 563 (1996), astro-ph/9611107.

[87] Arendt R.G., et al., ApJ. 508, 74 (1998). 
[88] Miyaji T., et al., astro-ph/9803320.

[89] Polyakov A.M. Sov. Phys. JETP 32, 296 (1971), Sov. Phys. JETP 33, 850 (1971), Koba Z., Nielsen H.B., \& Olesen P., Nucl. Phys. B 40, 317 (1972).

[90] Abalkonov V., et al., astro-ph/0001151. 

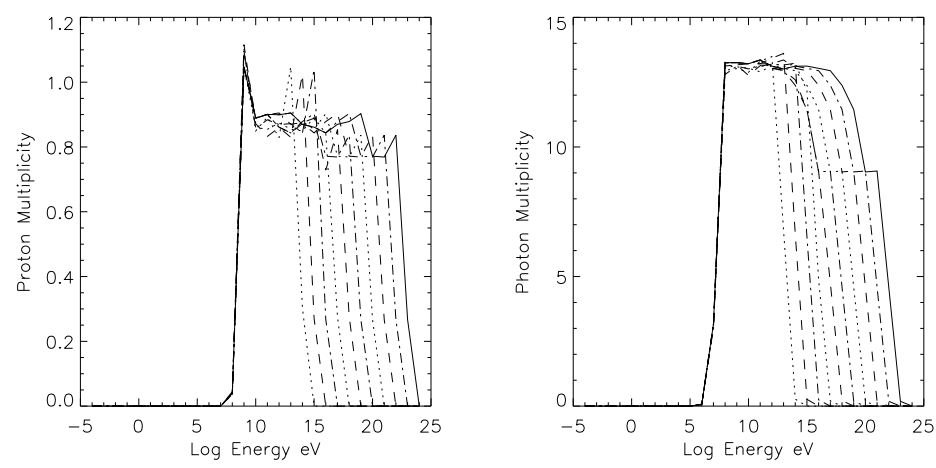

Figure 1: Proton and photon multiplicity in hadronization of a pair of gluon jets for $E_{C M}=$ $10^{14}-10^{24} \mathrm{eV}$. For $E_{C M}>10^{20} \mathrm{eV}$ the curves correspond to extrapolation from lower energies.

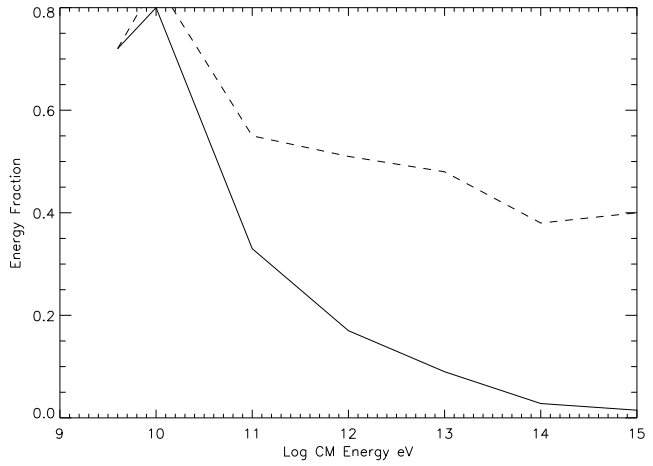

Figure 2: Energy fraction of leading proton (dashed line) and mean energy of protons (solid line) with respect to the CM energy in $p-\gamma$ interaction. 
Table 3: Interactions

\begin{tabular}{|c|c|c|c|c|c|}
\hline Interaction & $\begin{array}{l}\text { Analyt. Cal. } \\
E_{C M} \text { Rang }\end{array}$ & Cuts & $\begin{array}{c}\text { PYTHIA } E_{C M} \\
\text { Rang }\end{array}$ & PYTHIA Parameters & $\begin{array}{c}\text { Ref. Analytic } \\
\text { Cross-Sec. }\end{array}$ \\
\hline$e^{ \pm}-e^{ \pm}$elastic & All & $\begin{array}{c}\frac{s-4 m_{e}^{2}}{m_{e}^{2}}>10^{-3} \\
\left|\frac{t}{s}\right|>10^{-2},\left|\frac{u}{s}\right|>10^{-2}\end{array}$ & - & - & 72 \\
\hline$e^{+}-e^{-}$elastic & $E_{C M} \leq 4 G e V$ & $"$ & $\begin{array}{c}\text { included in } e^{+}-e^{-} \\
\rightarrow \ldots\end{array}$ & $\begin{array}{l}- \\
-\end{array}$ & $"$ \\
\hline$e^{+}-e^{-} \rightarrow 2 \gamma$ & $"$ & $"$ & $"$ & - & $"$ \\
\hline$e^{+}-e^{-} \rightarrow \ldots$ & - & - & $4 G e V \leq E_{C M} \leq 10^{6}$ & Init. Braamst. & - \\
\hline $\begin{array}{c}p^{ \pm}-e^{ \pm} \rightarrow \ldots \\
\text { (all combinations) }\end{array}$ & - & - & $"$ & - & - \\
\hline $\begin{array}{c}p^{ \pm}-p^{ \pm} \rightarrow \ldots \\
\text { (all combinations) }\end{array}$ & - & - & $3 G e V \leq E_{C M} \leq 10^{5}$ & - & - \\
\hline$\nu-e^{-}$elastic & All & $\frac{s-m_{e}^{2}}{m_{e}^{2}}>10^{-3}$ & - & - & 73 \\
\hline $\bar{\nu}-e^{+}$elastic & $"$ & $"$ & - & - & $"$ \\
\hline$\nu-e^{+} \rightarrow \ldots$ & - & - & $4 G e V \leq E_{C M} \leq 10^{6}$ & - & - \\
\hline $\bar{\nu}-e^{-} \rightarrow \ldots$ & - & - & $"$ & - & - \\
\hline$\nu-p^{ \pm} \rightarrow \ldots$ & - & - & $4 G e V \leq E_{C M} \leq 10^{6}$ & - & - \\
\hline $\bar{\nu}-p^{ \pm} \rightarrow \ldots$ & - & - & $"$ & - & - \\
\hline$\nu-\bar{\nu}$ & - & - & $"$ & - & - \\
\hline$\gamma-e \pm \rightarrow \gamma-e \pm$ & $E_{C M} \leq 4 G e V$ & $\begin{array}{c}\frac{s-m_{e}^{2}}{m_{e}^{2}}>10^{-3} \\
\left|\frac{t}{s}\right|>10^{-2},\left|\frac{u}{s}\right|>10^{-2}\end{array}$ & - & - & 72 \\
\hline$\gamma-e \pm \rightarrow \ldots$ & - & - & $4 G e V \leq E_{C M} \leq 10^{6}$ & - & - \\
\hline$\gamma-p \pm \rightarrow \ldots$ & $\begin{array}{c}1 G e V<E< \\
4 G e V\end{array}$ & - & $"$ & $\begin{array}{c}E_{C M}{ }^{\min }=1 \mathrm{GeV}, \\
p_{t}{ }^{\min }=0.5 \mathrm{GeV}, \\
\text { Singul. Cut }=0.25 \mathrm{GeV}\end{array}$ & 66 \\
\hline$\gamma-\gamma \rightarrow e^{+}-e^{-}$ & $E_{C M} \leq 3 G e V$ & $\begin{array}{c}\frac{s-m_{e}^{2}}{m_{e}^{2}}>10^{-3} \\
\left|\frac{t}{s}\right|>10^{-2},\left|\frac{u}{s}\right|>10^{-2}\end{array}$ & - & - & 72 \\
\hline$\gamma-\gamma \rightarrow \ldots$ & - & - & $3 G e V \leq E_{C M} \leq 10^{6}$ & - & - \\
\hline
\end{tabular}


Table 4: Energy flux of UHECRs close to GZK cutoff. Note that there is not a complete agreement between estimated flux by different Air Shower detectors. The values presented here are mostly based on AGASA data before 1998.

\begin{tabular}{|c|c|c|c|}
\hline $\log (E) \mathrm{eV}$ & $\begin{array}{c}\tau=5 \tau_{0}, \log E^{2} J(E) \\
m^{-2} \mathrm{sec}^{-1} s r^{-1}\end{array}$ & $\begin{array}{c}\tau=50 \tau_{0}, \log E^{2} J(E) \\
m^{-2} \mathrm{sec}^{-1} s r^{-1}\end{array}$ & $\begin{array}{c}\text { Observed log } E^{2} J(E) \\
m^{-2} \mathrm{sec}^{-1} s r^{-1}\end{array}$ \\
\hline 18.525 & 5.4 & 4.4 & $6.15 \pm 0.01$ \\
\hline 19.025 & 5.9 & 4.9 & $5.5 \pm 0.05$ \\
\hline 19.525 & 6.35 & 5.3 & $5.08 \pm 0.2$ \\
\hline 20.1 & 7 & 6 & $5.4 \pm 1.4$ \\
\hline 20.525 & 7.36 & 6.3 & $\sim 5 \pm 2$ \\
\hline 21.025 & 8.09 & 6.8 & \\
\hline
\end{tabular}
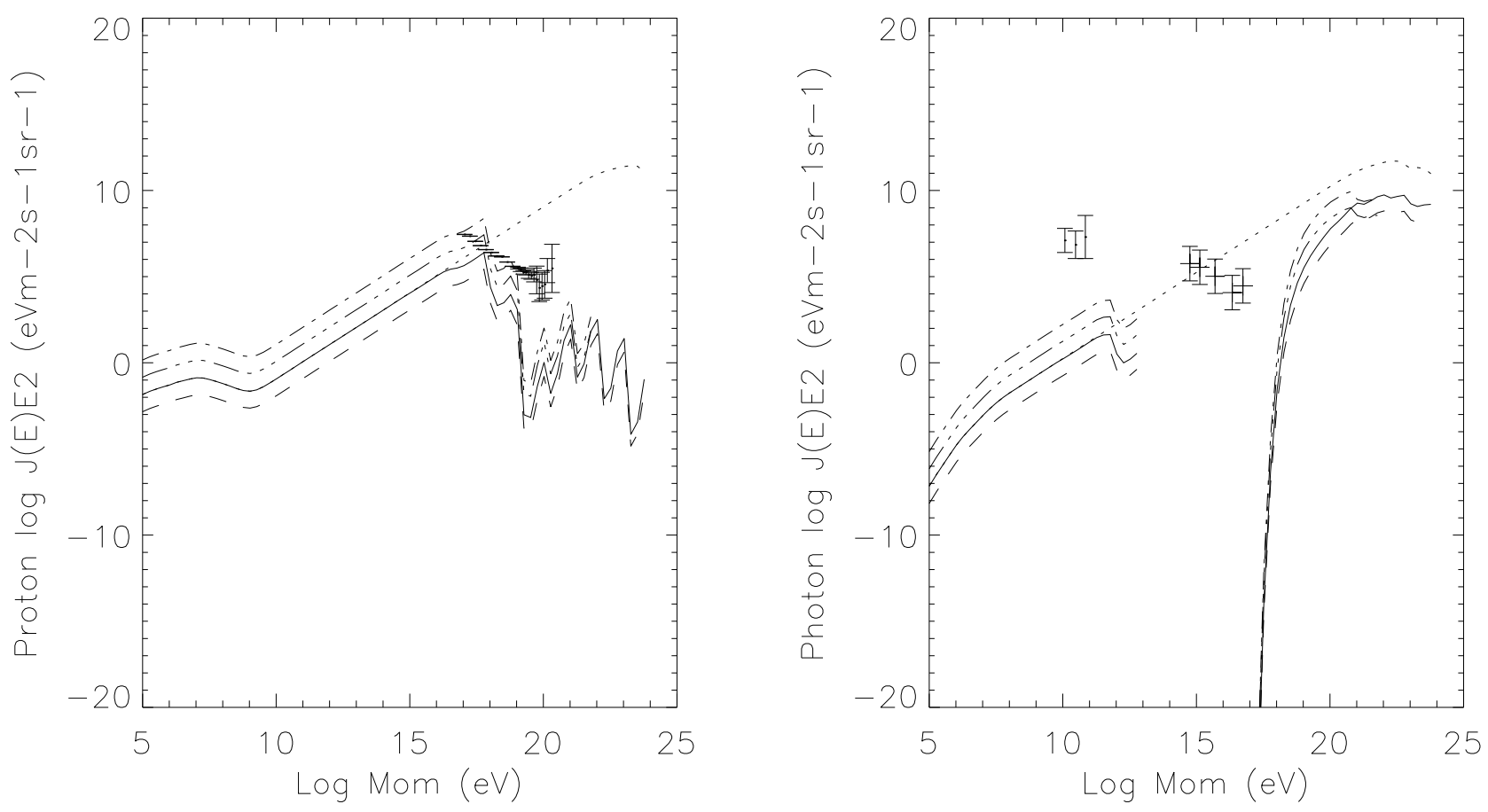

Figure 3: Energy flux for protons and photons. Solid line $m_{d m}=10^{24} \mathrm{eV}, \tau=5 \tau_{0}$, dot line is the spectrum without energy dissipation for the same mass and lifetime, dashed line $m_{d m}=10^{24} \mathrm{eV}$, $\tau=50 \tau_{0}$, dash $\operatorname{dot} m_{d m}=10^{22} \mathrm{eV}, \tau=5 \tau_{0}$, dash $\operatorname{dot} \operatorname{dot} \operatorname{dot} m_{d m}=10^{22} \mathrm{eV}, \tau=50 \tau_{0}$. For protons, data from Air Showers detectors [13] is shown. Data for photons are EGRET whole sky background [82] and upper limit from CASA-MIA [83. 

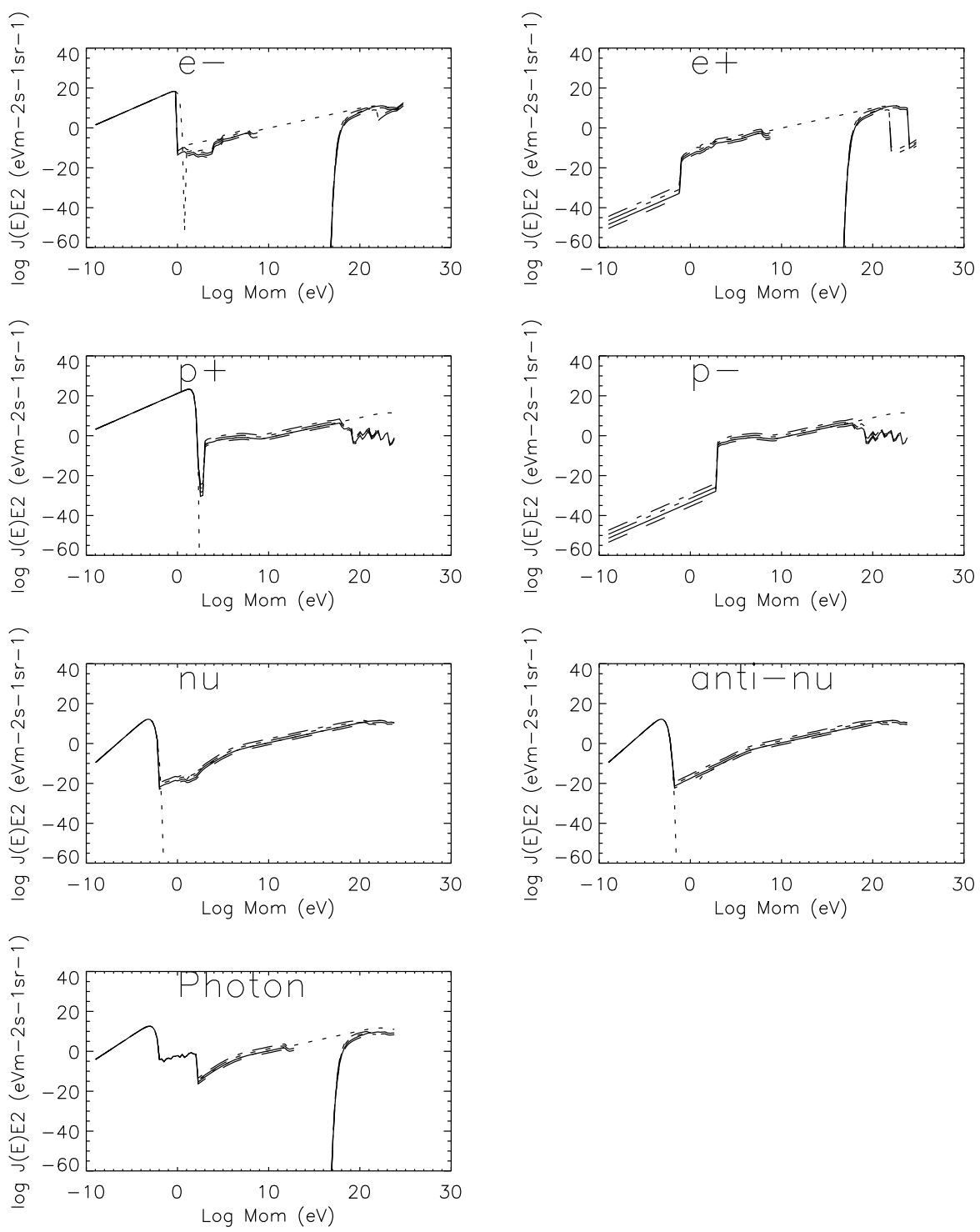

Figure 4: Energy flux of stable species. Description of curves is the same as Fig. 3 . 

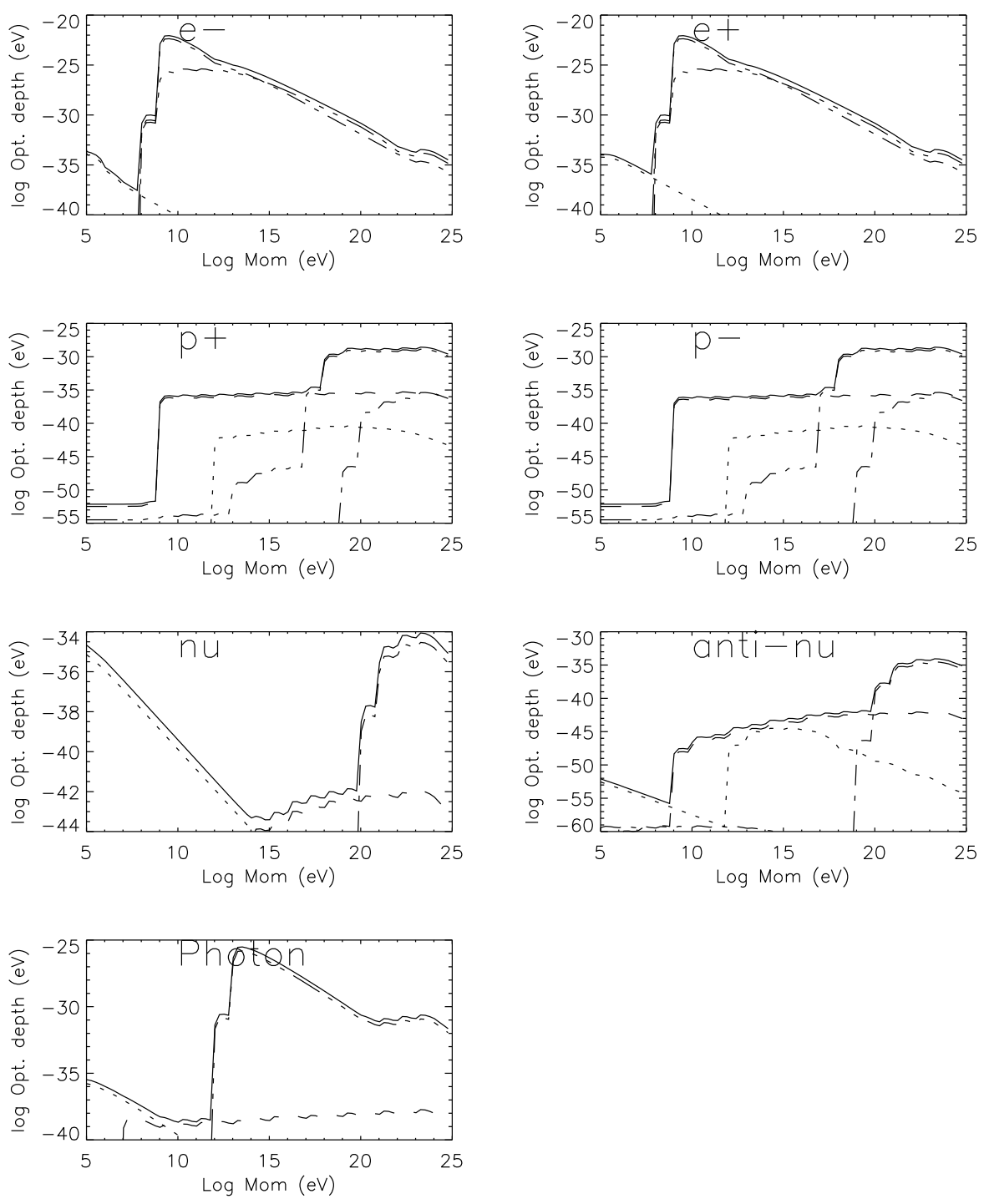

Figure 5: Total optical depth of species and contribution of backgrounds. Solid line is total optical depth, dot line contribution of $e^{ \pm}$, dashed line $p^{ \pm}$, dash dot $\nu \& \bar{\nu}$, and dash dot dot dot $\gamma$. Dependence on lifetime and mass of UHDM is negligible.

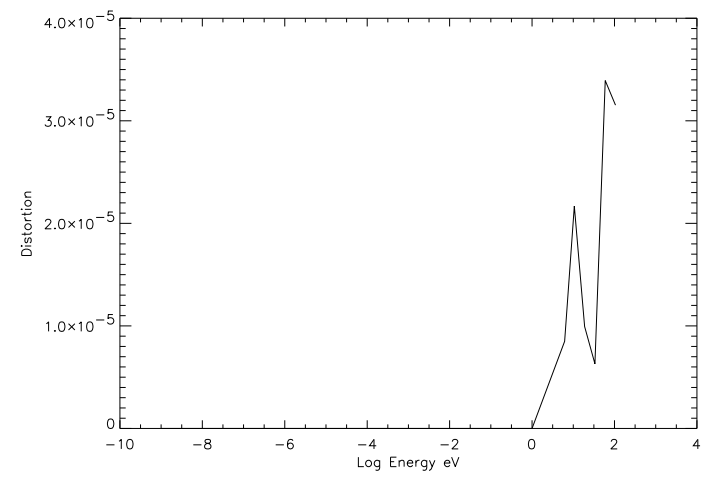

Figure 6: Fraction of distortion in photon distribution with respect to a stable DM. 


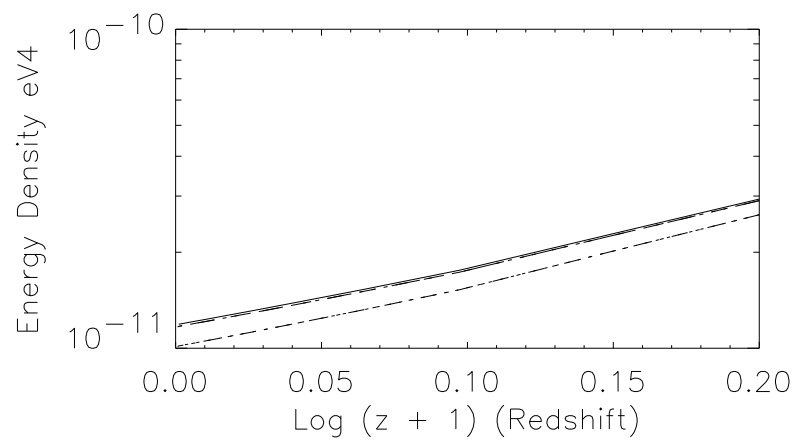

Figure 7: Energy density of the Universe. Solid line a stable DM, dash line $\tau=50 \tau_{0}$, dash dot $\tau=5 \tau_{0}$. Dependence on the mass of UHDM is negligible.
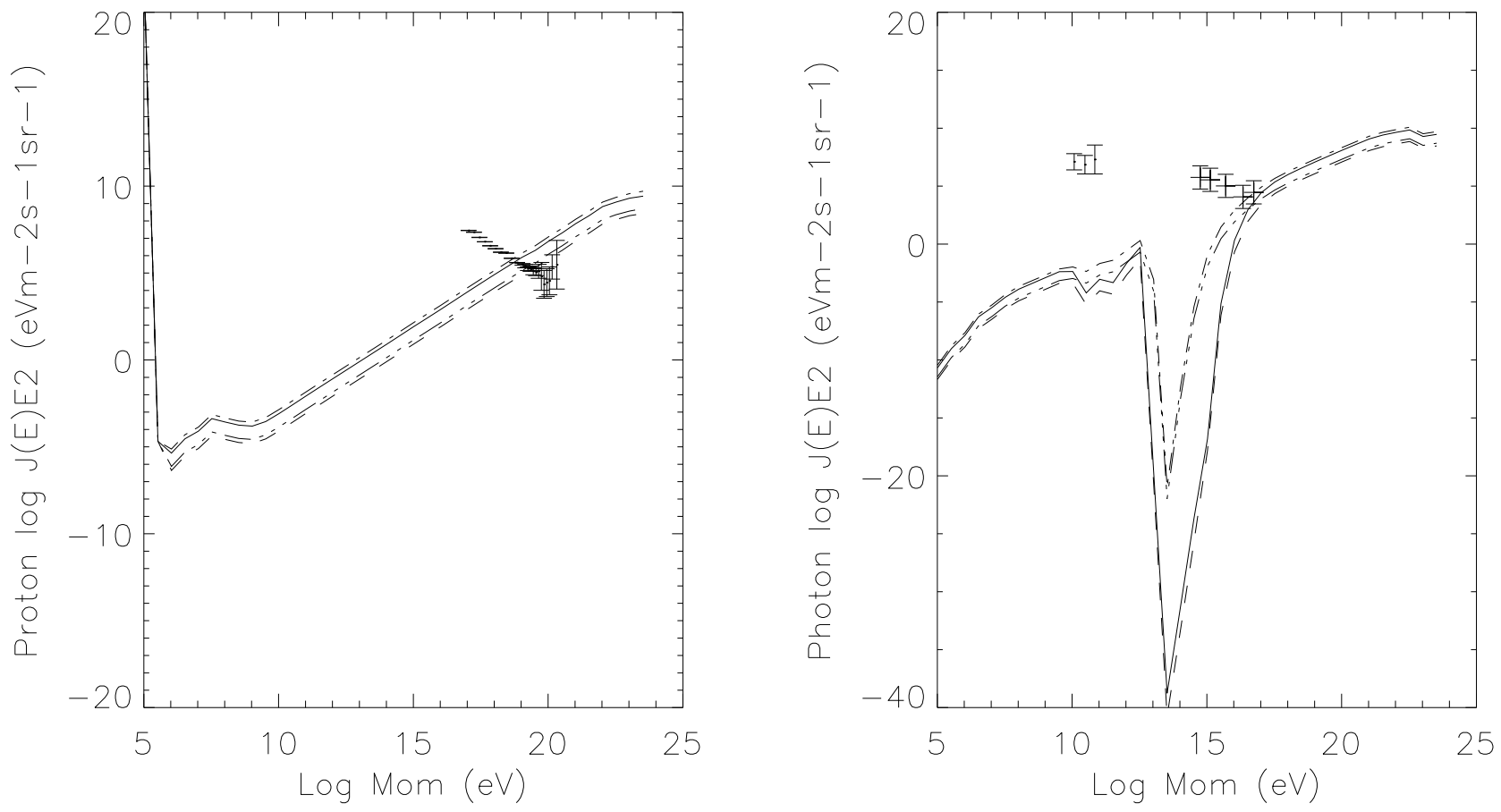

Figure 8: Flux of high energy protons and photons in a uniform clump. $m_{d m}=10^{24} \mathrm{eV}, \tau=5 \tau_{0}$ and $\tau=50 \tau_{0}$. Dash dot and dash dot dot dot lines presents UHDM halo. Solid and dashed lines show a halo of UHDM and MACHOs. Data is the same as in Fig. 3. For protons the effect of increasing lifetime of UHDM is more important than presence of MACHOs. Photons trough is more sensitive to presence of MACHOs. 


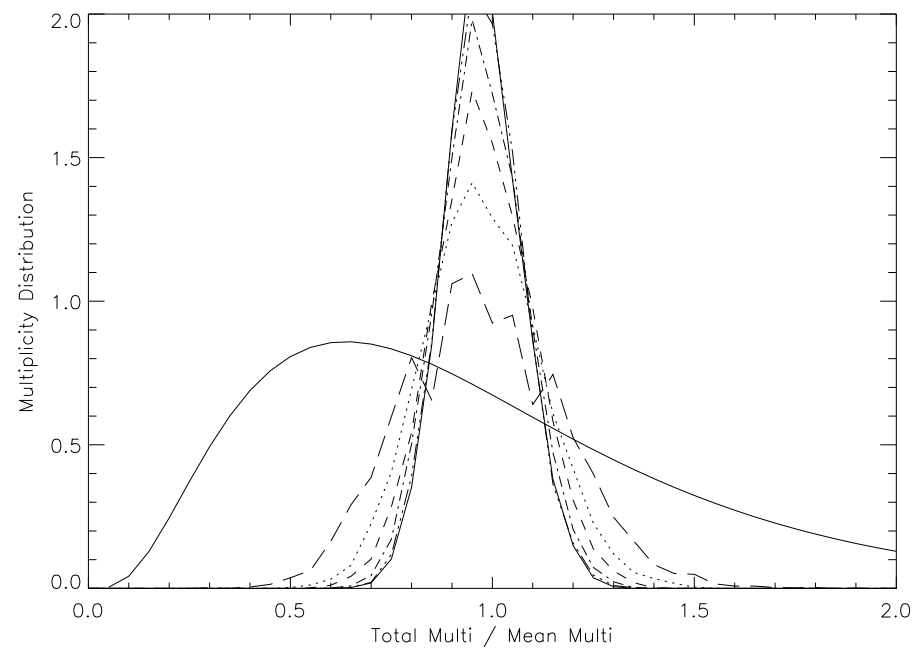

Figure 9: Distribution of multiplicity in hadronization of a pair of gluon jets. Solid line is the highest energy $E_{C M}=10^{20} \mathrm{eV}$; Long dash is lowest energy $E_{C M}=10^{11} \mathrm{eV}$. KNO distribution is shown also (solid line). 